\title{
Pacific
}

Journal of

Mathematics

\section{THE POSITIVE-DIMENSIONAL FIBRES OF THE PRYM MAP}

\author{
JUAN-CARLOS NARANJO
}

Volume $172 \quad$ No. 1 


\title{
THE POSITIVE DIMENSIONAL FIBRES OF THE PRYM MAP
}

\author{
JUAN-CARLOS NARANJO
}

\section{The fibres of positive dimension of the Prym map are char- acterized.}

Let $C$ be an irreducible complex smooth curve of genus $g$. Let $\pi: \tilde{C} \longrightarrow C$ be a connected unramified double covering of $C$.

The Prym variety associated to the covering is, by definition, the component of the origin of the Kernel of the norm map

$$
P(\tilde{C}, C)=\operatorname{Ker}\left(N m_{\pi}\right)^{0} \subset J \tilde{C} .
$$

It is a principally polarized abelian variety (p.p.a.v.) of dimension $g(\tilde{C})-g=$ $g-1$.

One defines the Prym map

$$
\begin{aligned}
& P_{g}: \quad \mathcal{R}_{g} \quad \longrightarrow \mathcal{A}_{g-1} \\
& (\tilde{C} \stackrel{\pi}{\rightarrow} C) \longmapsto P(\tilde{C}, C),
\end{aligned}
$$

where $\mathcal{R}_{g}$ is the coarse moduli space of the coverings $\pi$ as above and $\mathcal{A}_{g-1}$ stands for the coarse moduli space of p.p.a.v.'s of dimension $g-1$.

It is well-known that this map is generically injective for $g \geq 7$ (FriedmanSmith, Kanev). On the other hand this map is never injective; this is a consequence of the tetragonal construction due to Donagi (see [Do1] for a description of the construction). This fact is already implicit in the results of Mumford ([M]):

The coarse moduli space $\mathcal{R H}_{g}$ of unramified double coverings of smooth hyperelliptic curves of genus $g$ has $\left[\frac{g-1}{2}\right]+1$ irreducible components $\mathcal{R H}_{g, t}$, $t=0, \ldots,\left[\frac{g-1}{2}\right]$. For an element $(\tilde{C}, C) \in \mathcal{R H}_{g, t}$ there exist two hyperelliptic curves

$$
p_{1}: C_{1} \longrightarrow \mathbb{P}^{1}, \quad p_{2}: C_{2} \longrightarrow \mathbb{P}^{1}
$$

of genus $g\left(C_{1}\right)=t \leq g-t-1=g\left(C_{2}\right)$ such that

a) $\tilde{C}=C_{1} \times \mathbb{P}^{1} C_{2}$ and

b) $C=\tilde{C} /\left(\sigma_{1} \circ \sigma_{2}\right)$, where $\sigma_{1}$ (resp. $\left.\sigma_{2}\right)$ is the involution on $\tilde{C}$ attached to the branched covering $\tilde{C} \longrightarrow C_{1}$ (resp. $\tilde{C} \longrightarrow C_{2}$ ). 
Mumford proves (loc. cit. p. 346) that one has an isomorphism of p.p.a.v.

$$
P(\tilde{C}, C) \cong J C_{1} \times J C_{2} .
$$

Consequently the fibres of the restriction of $P_{g}$ to $\mathcal{R} \mathcal{H}_{g}$ have positive dimension. In fact $P_{g}\left(\mathcal{R} \mathcal{H}_{g, t}\right)$ is contained in the product $\mathcal{J H}_{t} \times \mathcal{J H}_{g-t-1}$, where $\mathcal{J H}_{s}$ stands for the locus of Jacobians of hyperelliptic curves of genus $s$. Thus

$$
\operatorname{dim} \mathcal{R H}_{g, t}=2 g-1>\operatorname{dim} \mathcal{J H}_{t} \times \mathcal{J H}_{g-t-1}= \begin{cases}2 g-4 & \text { if } t \neq 0 \\ 2 g-3 & \text { if } t=0 .\end{cases}
$$

On the other hand positive dimensional fibres also appear for some coverings of bi-elliptic curves (a curve is called bi-elliptic if it can be represented as a ramified double covering of an elliptic curve).

In this note we characterize the fibres of positive dimension of the Prym map. To state our theorem we need some notation: let $\mathcal{R} \mathcal{B}_{g}$ be the coarse moduli space of the unramified double coverings $\pi: \tilde{C} \longrightarrow C$ such that $C$ is a smooth bi-elliptic curve of genus $g$. This variety has $\left[\frac{g-1}{2}\right]+2$ irreducible components

$$
\mathcal{R B}_{g}=\left(\bigcup_{t=0}^{\left[\frac{g-1}{2}\right]} \mathcal{R} \mathcal{B}_{g, t}\right) \cup \mathcal{R} \mathcal{B}_{g}^{\prime}
$$

(see $[\mathbf{N}]$ for more details).

We obtain:

Theorem. Assume $g \geq 13$. A fibre of $P_{g}$ is positive dimensional at $(\tilde{C}, C)$ if and only if $C$ is either hyperelliptic or

$$
(\tilde{C}, C) \in \bigcup_{t \geq 1} \mathcal{R B}_{g, t}
$$

Proof. If $C$ is hyperelliptic we apply the results of Mumford. On the other hand, all the irreducible components of the fibres of $P_{g \mid \mathcal{R} \mathcal{B}_{g, t}}$ are positive dimensional for $t \geq 1$ (see [N, $\S 20]$ ). This finishes one implication.

The first step to see the opposite implication is to prove that the curve $C$ is tetragonal (i.e. there exists a $g_{4}^{1}$ on $C$ ).

Let $\eta \in J C$ be the two-torsion point characterizing the covering and denote by $L$ the line bundle $\omega_{C} \otimes \eta$. It is easy to check that $L$ is very ample if $C$ is non-tetragonal. Let $\Phi_{L}$ be the projective embedding of $C$ defined by $L$.

As in Beauville ([B, p. 379]), we replace $\mathcal{R}_{g}$ and $\mathcal{A}_{g-1}$ by the corresponding functors. Then, the Prym map defines a morphism of functors $P r_{g}$. Our 
hypothesis on the fibre of $P_{g}$ implies that the cotangent map to $\operatorname{Pr}_{g}$ at $(\tilde{C}, C)$ is not surjective. By loc. cit. Prop. (7.5), this map can be shown as the cup-product map

$$
S^{2} H^{0}(C, L) \longrightarrow H^{0}\left(C, L^{\otimes 2}\right)
$$

followed by the isomorphism induced in cohomology by $L^{\otimes 2} \cong \omega^{\otimes 2}$. Hence, the non-surjectivity implies that $\Phi_{L}(C)$ is not a projectively normal curve.

We recall Theorem 1 in [G-L]: If $L$ is very ample and

$$
\operatorname{deg}(L) \geq 2 g+1-2 h^{1}(L)-\operatorname{Cliff}(C),
$$

then $\Phi_{L}(C)$ is projectively normal (where $\operatorname{Cliff}(C)$ is the Clifford index of $C)$.

Since $h^{1}(L)=0$ and $\operatorname{deg}(L)=2 g-2$ one obtains $\operatorname{Cliff}(C) \leq 2 . \quad$ By using Clifford's Theorem and [Ma, Propositions 7 and 8], it follows that the curve either possess a $g_{4}^{1}$ or is plane curve of degree six. The second case contradicts $g \geq 13$.

Thus $C$ is tetragonal. Since $g \geq 13$ the results in $[\mathbf{D e}]$ can be applied: either the fibre is finite (generically, three elements) or we are in one of the following three possibilities: $C$ is either hyperelliptic or bi-elliptic or trigonal.

Assume that $C$ is bi-elliptic. Theorems (9.4), (10.9) and (10.10) in [N] states that $P_{g}^{-1}(P(\tilde{C}, C))$ consists of two points for every $(\tilde{C}, C) \in \mathcal{R B}_{g, 0} \cup$ $\mathcal{R B}_{g}^{\prime}$, hence

$$
(\tilde{C}, C) \in \bigcup_{t \geq 1} \mathcal{R B}_{g, t}
$$

To finish the proof we have to rule out the case: $C$ trigonal. In $[\mathbf{R}]$, Recillas (cf. also [Do2]) establishes an isomorphism

$$
\tau: \mathcal{R} \mathcal{T}_{g} \cong \mathcal{M}_{g-1}^{\text {tet, } 0}
$$

where $\mathcal{R} \mathcal{T}_{g}$ is the coarse moduli space of unramified double coverings of trigonal curves and $\mathcal{M}_{g-1}^{t e t, 0}$ is the moduli space of pairs $\left(X, g_{4}^{1}\right)$ of tetragonal curves $X$ and a base-point-free tetragonal linear series on $X$ not containing divisors of the form $2 x+2 y$. This map satisfies that

$$
\tau(\tilde{C}, C)=\left(X, g_{4}^{1}\right) \Longrightarrow P(\tilde{C}, C) \cong J X \quad \text { (as p.p.a.v.). }
$$

Let us fix $(\tilde{C}, C)$ as above and let $(\tilde{D}, D) \in \mathcal{R}_{g}$ such that $P(\tilde{D}, D) \cong$ $P(\tilde{C}, C) \cong J X$. Since $C$ is not hyperelliptic, then the singular locus of the theta divisor of $P(\tilde{D}, D)$ has codimension 3 by [M, p. 344]. In loc. cit. a list of the Prym varieties with such property appear. We obtain that $D$ is either trigonal or bi-elliptic. Since $P(\tilde{D}, D)$ is the Jacobian of a curve the bi-elliptic case contradicts $[\mathbf{S}]$. 
Hence it suffices to prove that all the fibres of the restriction of $P_{g}$ to $\mathcal{R} \mathcal{T}_{g}$ are zero dimensional. This follows from the bijection $\tau$. Indeed, a curve $X$ of genus $g \geq 12$ has at most one base-point-free $g_{4}^{1}$ without divisors of the form $2 x+2 y$; otherwise there exists a map $f: X \longrightarrow \mathbb{P}^{1} \times \mathbb{P}^{1}$ and then either the genus is $\leq 9$ or $X$ is bi-elliptic. By [ $\mathbf{T}$, Lemma (4.3)] the linear series of degree 4 and dimension 1 on a bi-elliptic curve come from $g_{2}^{1}$ linear series on the elliptic curve, thus divisors of the forbidden form appear.

Now the classical Torelli Theorem says that

$$
\begin{aligned}
\mathcal{M}_{g-1}^{t e t, 0} & \longrightarrow \mathcal{A}_{g-1} \\
\left(X, g_{4}^{1}\right) & \longmapsto J X
\end{aligned}
$$

is injective. Composing with $\tau$ we are done.

Remark. Note that if one drops the hypothesis on the genus, at least one gets that the Clifford index of $C$ is $\leq 2$.

\section{References}

[B] A. Beauville, Variétés de Prym et Jacobiennes Intermédiaires, Ann. Sci. E.N.S., 10 (1977), 309-391.

[De] O. Debarre, Sur les variétés de Prym des courbes tétragonales, Ann. Sci. E.N.S., 21 (1988), 545-559.

[Do1] R. Donagi, The tetragonal construction, Bull. Amer. Math. Soc., 4 (1981), 181-185.

[Do2] R. Donagi, The fibres of the Prym map, Preprint 1992.

[G-L] M. Green and R. Lazarsfeld, On projective normality of complete linear systems on an algebraic curve, Invent. Math., 83 (1985), 73-90.

[M] D. Mumford, Prym varieties, I, in Contributions to Analysis, New York 1974.

[Ma] G. Martens, Funktionen von vorgegebener Ordnung auf Komplexen Kurven, J. reine angew. Math., 320 (1980), 68-85.

[N] J.C. Naranjo, Prym varieties of bi-elliptic curves, J. reine angew. Math., 424 (1992), 47-106.

[R] S. Recillas, Jacobians of curves with $g_{4}^{1}$ 's are the Prym's of trigonal curves, Bol. Soc. Mat. Mexicana, 19 (1974), 9-13.

[S] V.V. Shokurov, Distinguishing Prymians from Jacobians, Invent. Math., 65 (1981), 209-219.

[T] M. Teixidor, On translation invariance for $W_{d}^{r}$, J. reine angew. Math., 385 (1988), 10-23.

Received March 1, 1993. The author was partially supported by the European Science Program, "Geometry of Algebraic Varieties" project, contract no. SC1-0398-C(A) and by the DGICYT no. PS90-0069.

Departament D'Algebra i Geometria

Universitat de Barcelona, Gran Via 585

08007 BARCELONA, SPAIN

E-mail address: naranjo@cerber.ub.es 



\section{PACIFIC JOURNAL OF MATHEMATICS}

Volume $172 \quad$ No. $1 \quad$ January 1996

A class of incomplete non-positively curved manifolds

BRIAN BOWDITCH

The quasi-linearity problem for $C^{*}$-algebras

41

L. J. BUNCE and JOHN DAVID MAITLAND WRIGHT

Distortion of boundary sets under inner functions. II

Jose Luis Fernandez Perez, Domingo Pestana and José Rodríguez

Irreducible non-dense $A_{1}^{(1)}$-modules

VJACHESLAV M. FUTORNY

$M$-hyperbolic real subsets of complex spaces

101

Giuliana Gigante, Giuseppe Tomassini and Sergio Venturini

Values of Bernoulli polynomials

ANDREW GRANVILLE and ZHI-WEI SUN

The uniqueness of compact cores for 3-manifolds

LUKE HARRIS and PETER SCOTT

Estimation of the number of periodic orbits

BOJU JIANG

Factorization of $p$-completely bounded multilinear maps

Christian Le MERdy

Finitely generated cohomology Hopf algebras and torsion

JAMEs Peicheng LiN

The positive-dimensional fibres of the Prym map

JUAN-CARLOS NARANJO

Entropy of a skew product with a $Z^{2}$-action

KYEWON KOH PARK

Commuting co-commuting squares and finite-dimensional Kac algebras

TAKASHI SANO

Second order ordinary differential equations with fully nonlinear two-point boundary 255 conditions. I

H. BEVAN THOMPSON

Second order ordinary differential equations with fully nonlinear two-point boundary conditions. II

H. BEVAN THOMPSON

The flat part of non-flat orbifolds

FENG XU 